\title{
BSA Nanoparticles for siRNA Delivery: Coating Effects on Nanoparticle Properties, Plasma Protein Adsorption, and In Vitro siRNA Delivery
}

\author{
Haran Yogasundaram, 1, 2 Markian Stephan Bahniuk, ,, 2 Harsh-Deep Singh, ${ }^{1}$ \\ Hamidreza Montezari Aliabadi, ${ }^{1}$ Hasan Uludağ, ${ }^{1,3}$ and Larry David Unsworth ${ }^{1,2,3}$ \\ ${ }^{1}$ Department of Chemical and Materials Engineering, Faculty of Engineering, University of Alberta, Edmonton, AB, Canada T6G 2G6 \\ ${ }^{2}$ National Research Council, National Institute for Nanotechnology, Edmonton, AB, Canada T6G 2M9 \\ ${ }^{3}$ Department of Biomedical Engineering, Faculty of Medicine and Dentistry, University of Alberta, Edmonton, AB, Canada T6G 2 G6
}

Correspondence should be addressed to Hasan Uludağ, huludag@ualberta.ca and Larry David Unsworth, lunswort@ualberta.ca

Received 12 April 2012; Accepted 21 June 2012

Academic Editor: Esmaiel Jabbari

Copyright ( 2012 Haran Yogasundaram et al. This is an open access article distributed under the Creative Commons Attribution License, which permits unrestricted use, distribution, and reproduction in any medium, provided the original work is properly cited.

\begin{abstract}
Developing vehicles for the delivery of therapeutic molecules, like siRNA, is an area of active research. Nanoparticles composed of bovine serum albumin, stabilized via the adsorption of poly-L-lysine (PLL), have been shown to be potentially inert drugdelivery vehicles. With the primary goal of reducing nonspecific protein adsorption, the effect of using comb-type structures of poly(ethylene glycol) ( $1 \mathrm{kDa}, \mathrm{PEG}$ ) units conjugated to PLL ( 4.2 and $24 \mathrm{kDa}$ ) on BSA-NP properties, apparent siRNA release rate, cell viability, and cell uptake were evaluated. PEGylated PLL coatings resulted in NPs with $\zeta$-potentials close to neutral. Incubation with platelet-poor plasma showed the composition of the adsorbed proteome was similar for all systems. siRNA was effectively encapsulated and released in a sustained manner from all NPs. With $4.2 \mathrm{kDa}$ PLL, cellular uptake was not affected by the presence of PEG, but PEG coating inhibited uptake with $24 \mathrm{kDa}$ PLL NPs. Moreover, $24 \mathrm{kDa}$ PLL systems were cytotoxic and this cytotoxicity was diminished upon PEG incorporation. The overall results identified a BSA-NP coating structure that provided effective siRNA encapsulation while reducing $\zeta$-potential, protein adsorption, and cytotoxicity, necessary attributes for in vivo application of drugdelivery vehicles.
\end{abstract}

\section{Introduction}

Short interfering RNA (siRNA) is extremely promising for the therapeutic treatment of a myriad of diseases; however, its clinical application has hitherto been hindered by an apparent inability to control its delivery. The use of NP based drug delivery vehicles presents several advantages over conventional delivery stratagems, including the fact that they may be used for precise tissue targeting, remain in blood for a prolonged time, and be immediately injected into the systemic circulation. Furthermore, favorable tissue responses have been observed for decreasing particle sizes [1] and a multitude of covalent and noncovalent modifications of NP surfaces can be achieved, aspects that facilitate the design of more effective carriers. In particular, BSA-based NPs have many advantageous qualities [2]: presence of a hydrophobic core facilitating delivery of hydrophobic drugs, a natural abundance in plasma, relative stability and inertness in biochemical pathways, availability, and a relatively benign in vivo biological fate [3]. Unlike NPs fabricated from synthetic polymers, it is thought that the natural protein removal mechanisms will result in a reduced overall toxicity related to the application of BSA NPs [3]. That said, an important step in facilitating the localization of these NPs at the site of interest involves both decreasing their removal from the circulation (i.e., decreasing opsonization) as well as ensuring that any targeting moiety remains able to interact with the cellular site of interest. Inhibiting nonspecific protein adsorption will then be central to both of these effects. A common strategy for preventing protein adsorption at 
the tissue-material interface is to incorporate end-tethered PEG to the surfaces of biomaterials. It has been well established that the presence of end-tethered PEG can prevent particulate aggregation, reduce interactions with plasma proteins [4], minimize reticuloendothelial system clearance, and prolong blood circulation time of a host of NPs [5-7].

PEGylation of surfaces has been shown to impede nonspecific protein adsorption [8], where both the presence and conformation of end-tethered PEG play a critical role $[9,10]$. It is noteworthy that not only is the amount of plasma protein adsorbed at the tissue-biomaterial interface important in obfuscating an engineered surface but also the composition of the protein layer itself is critical, as this may ultimately direct host responses. NP opsonization has been correlated to surface properties, including hydrophilicity, roughness, $\zeta$-potential, and surface chemistry [11]. Recent results [12] have shown that systemic administration of BSA NPs, stabilized with polyethyleneimine-graft-PEG with bisphosphonic acid attached for bone targeting, showed no beneficial effects associated with the polymer coating. Although the reason for this result was not fully elucidated, it was postulated that the biodistribution of the NPs may be affected by the presence of the adsorbed protein corona to the PEG modified NPs; it is hoped that further analyzing the adsorbed protein composition to these PEG modified BSA NPs may clarify this point. Other previous work [13] has looked specifically at the use of positively charged polyL-lysine (PLL) as a coating polymer that stabilizes the NP used for the apparent release of siRNA from BSA NPs. It was observed that, for low concentrations of PLL, varying the size of the PLL used for coating resulted in minimal effect on the net release of siRNA from the NPs. In further work [14], the release of a model drug from BSA NPs could be controlled from $\sim 5$ to $90 \%$ over 14 days, depending on the nature of coating designed to display differential stability against endogenous enzymes.

In this study, we continued the development of BSA NPs by exploring the role of PEG coating by employing comblike structures of PEG-conjugated PLLs and compared systems stabilized via unmodified PLL. Specifically, PLLs of 4.2 and $24 \mathrm{kDa}$ were utilized to understand the effect molecular weight may have on critical issues related to NP stabilization, siRNA encapsulation and passive release kinetics, plasma protein adsorption, cytotoxicity, and cellular incorporation. Conjugates of PLLs with $1 \mathrm{kDa}$ PEG were synthesized so as to determine if any direct effect on NP stabilization as well as siRNA encapsulation and passive release kinetics might be altered. The cellular uptake of the NPs and the plasma protein adsorption profile were assessed, investigating the role of PEG coating on these features. Our results identified specific types of BSA NPs that provided adequate siRNA release and cellular uptake with relatively low amounts of protein adsorption and cytotoxicity.

\section{Materials and Methods}

BSA and $\mathrm{HBr}$ salt of PLLs of different MWs (4.2 and $24 \mathrm{kDa}$ ) were purchased from Sigma-Aldrich (St. Louis, MO, USA) and used without further purification. The sodium dodecyl sulfate (SDS) was obtained from J. T. Baker (Phillipsburg, NJ, USA). FAM-labelled siRNA (double stranded, 21 base pairs) was purchased from Ambion Inc. (Austin, TX, USA). EDTA/trypsin (10X; Invitrogen, Carlsbad, CA, USA) was diluted 1:10 with Hank's Buffered Salt Solution (HBSS; Invitrogen) to $0.05 \mathrm{~g} / \mathrm{L}$ concentration before use. Dulbecco's Modified Eagle Medium (DMEM; high glucose), and penicillin/streptomycin $(10000 \mathrm{U} / \mathrm{mL} / 10 \mathrm{mg} / \mathrm{mL})$ solution were obtained from Invitrogen. Fetal bovine serum (FBS) was from PAA Laboratories (Etobicoke, Ontario, Canada). Sodium phosphate, monobasic, monohydrate sodium phosphate, and sodium chloride laboratory-grade reagents were purchased from EMD Chemical Inc. (Darmstadt, Germany). Ethanol was purchased from Fischer Scientific (Ottawa, Ontario, Canada). The N-hydroxysuccinimide ester of $1 \mathrm{kDa}$ (mPEG-NHS) was obtained from Creative PEG works, NC, USA. The 3,3',5,5'-tetramethylbenzidine substrate (TMBS) was obtained from Promega (Madison, WI, USA). The dialysis tubing of various MW cutoffs was obtained from Spectrum Laboratories Inc. (Rancho Dominguez, CA, USA).

2.1. PEG Conjugation to PLL. Previously reported methods were used to conjugate PLL to $1 \mathrm{kDa}$ PEG [15]. Briefly, PLL was dissolved in $0.1 \mathrm{M}$ phosphate buffer $(\mathrm{PB}, \mathrm{pH} 7.4)$ to a final concentration of $2.4 \mathrm{mg} / \mathrm{mL}$. mPEG-NHS was diluted to $18 \mathrm{mM}$ using $0.1 \mathrm{M} \mathrm{PB}$, added to the PLL solution, and reacted for $3 \mathrm{hrs}$ at room temperature. Solution was dialyzed 2 days against MilliQ $(18 \Omega)$ water, at $4^{\circ} \mathrm{C}$; MilliQ water being changed twice a day. Dialysate containing 4.2 and $24 \mathrm{kDa}$ PLL was freeze-dried and used to reconstitute the polymer solutions at desired concentrations.

2.2. NP Preparation. BSA NPs were formed via a coacervation method as detailed elsewhere [13]. Briefly, $250 \mu \mathrm{L}$ of $10 \mathrm{mg} / \mathrm{mL}$ BSA in water was added dropwise to an equal volume of $10 \mathrm{mM} \mathrm{NaCl}$ in water at room temperature. After $15 \mathrm{~min}$ stirring (600 rpm, room temperature), ethanol was added dropwise to a final volume ratio of $6: 1$, ethanol to BSA solution. The mixture was stirred for $3 \mathrm{hrs}$ to form NPs. To stabilize formed NPs, native PLLs $(0.3 \mathrm{mg} / \mathrm{mL})$ or PEGconjugated PLLs ( $0.3 \mathrm{mg}$ PLL equivalents $/ \mathrm{mL}$ ) in deionized water were introduced dropwise to the equal volume of NPs suspension under constant stirring. Stirring was continued for $1 \mathrm{hr}$ at room temperature to ensure time for the PLL and PEG-PLL conjugates to adsorb to the BSA NP surface. Suspensions of coated NPs were dialyzed against $\mathrm{ddH}_{2} \mathrm{O}$ for 3 days ( $12 \mathrm{hrs}$ between solution replacements). Where indicated, the amount of coating on the surface was determined by using the FITC-labeled PLL or PEG-PLL. The polymer labeling was achieved by incubating $10 \mu \mathrm{L}$ of $0.1 \mathrm{mM}$ FITC solution (in DMSO) with $1 \mathrm{~mL}$ of PLL or PEG-PLL $(2 \mathrm{mg} / \mathrm{mL}$ in $100 \mathrm{mM}$ phosphate buffer, $\mathrm{pH}=7.4$ ) for $1 \mathrm{hr}$ at room temperature. Ethanol $(9 \mathrm{~mL})$ was then added to this solution. The solution then was centrifuged at room temperature $(3000 \mathrm{rpm})$ for 15 minutes, and the supernatant, with unconjugated FITC, was removed. The pellet formed during this process was further washed with $5 \mathrm{~mL}$ of ethanol 
and centrifuged at $3000 \mathrm{rpm}$ for $15 \mathrm{~min}$ [13]. The solids obtained were air-dried under vacuum for $5 \mathrm{hrs}$ and stored in the dark at $4^{\circ} \mathrm{C}$ until used. The coating of FITC-labeled polymer was determined using fluorescence measurements. The coated NPs suspension was then diluted with phosphate buffer $(\mathrm{pH}=7.4)$ by $100 \%$ and centrifuged $(15,000 \mathrm{rpm}$, $1 \mathrm{hr})$ and fluorescence $\left(\lambda_{\mathrm{EX}}: 485 \mathrm{~nm} ; \lambda_{\mathrm{EM}}: 527 \mathrm{~nm}\right)$ of the supernatant was analyzed using a multiwall plate reader (Thermo Labsystems, Franklin, MA, USA). A calibration curve generated was used to calculate the coating efficiency as (1 - FITC-polymer supernatant $_{\text {(FITC-polymer }}$ supernatant + FITC-polymer pellet $)) \times 100 \%$.

2.3. Particle Sizing and $\zeta$-Potential. Mean particle size of the coated and uncoated NPs were determined using dynamic light scattering (Zetasizer $3000 \mathrm{HS}$, Malvern Instruments Ltd., UK) with a $633 \mathrm{~nm} \mathrm{He}-\mathrm{Ne}$ laser at a scattering angle of $90^{\circ}$. Uncoated BSA NPs were used directly for the measurements while the coated BSA NPs were diluted $1: 2$ with $\mathrm{PB}(10 \mathrm{mM}, \mathrm{pH} 7.4)$. Intensity measurements were used to determine the NP size. The $\zeta$-potential of the NPs was determined by measuring their electrophoretic mobility using the same instrument at $25^{\circ} \mathrm{C}$.

2.4. Plasma Incubation and Elution. Human blood plasma was obtained from Canadian Blood Services, where Canadian Blood Services obtained written informed consent from all volunteers for the collection and distribution of human blood products for research purposes. Human blood products were then shipped to our lab and used following research ethics procedures as approved by the University of Alberta Research Ethics Board (Institutional), Canadian Blood Services Research Ethics Board (Federal), and the National Research Council of Canada Research Ethics Board (Federal). NP solutions $(500 \mu \mathrm{L})$ were combined with an equal volume of undiluted human plasma and incubated at room temperature for 2 hrs with gentle rocking. The samples were then centrifuged at $13000 \mathrm{rpm}$ for 10 minutes and the supernatants discarded. Pellets were resolubilized in $1 \mathrm{~mL}$ of $0.15 \mathrm{M}$ phosphate buffered saline (PBS; $\mathrm{pH} 7.4$ ) for 30 minutes and the procedure was repeated two times. These samples were spun down and pellets were solubilized in $2 \%$ $\mathrm{w} / \mathrm{v}$ SDS in $0.15 \mathrm{M}$ PBS for one hr, with rocking at room temperature in order to elute adsorbed plasma proteins. To separate the NPs from the eluted plasma proteins, the samples were centrifuged at $13000 \mathrm{rpm}$ for 10 minutes and the supernatants collected and characterized using SDSPAGE and immunoblotting analysis.

2.5. SDS-PAGE and Immunoblotting. Reduced SDS-PAGE and immunoblotting techniques were used to evaluate and to identify eluted proteins as described previously [9]. All electrophoretic apparatus were purchased from Bio-Rad (Hercules, CA). Briefly, samples were separated on 12\% SDSPAGE gels and then transferred onto a $0.2 \mu \mathrm{m}$ immunoblot PVDF membrane. The membrane was cut into strips for total protein staining using colloidal gold (Bio-Rad) and for immunoblotting. Primary and secondary antibodies (see
Table S1 in Supplementary Materials available online at doi:10.1155/2012/584060) were used without further purification at concentrations of $1: 1000$. To visualize proteinantibody complexes, $350 \mu \mathrm{L}$ of stabilized TMBS substrate was incubated with membrane strips for 10 minutes at room temperature, with rocking. The colour-developing reaction was then quenched for 10 minutes using $2 \mathrm{~mL}$ of MilliQ water.

\subsection{Preparation of siRNA Loaded BSA NPs and Release Stud-} ies. The coacervation technique previously described was employed to prepare BSA NPs encapsulating siRNA. Briefly, $500 \mu \mathrm{L}$ of aqueous solution of BSA $(10 \mathrm{mg} / \mathrm{mL})$ was added to an equal volume of $10 \mathrm{mM} \mathrm{NaCl}$ solution under stirring $(600 \mathrm{rpm})$ in glass vials. The stirring was continued for 15 minutes at room temperature. To achieve siRNA encapsulation, siRNA solution $(20 \mu \mathrm{L}$ of $0.15 \mathrm{mg} / \mathrm{mL})$ was added to this binary solution and stirred at $600 \mathrm{rpm}$ for $1 \mathrm{hr}$ at room temperature. The siRNA (scrambled) used for encapsulation was either unlabeled or labeled with FAM. NPs were then formed by adding ethanol dropwise (final volume ratio of ethanol to starting BSA solution $=6$ ). Stirring was continued for $3 \mathrm{hrs}$ at room temperature after the complete addition of ethanol. To coat these siRNA loaded BSA NPs with PLL's, $500 \mu \mathrm{L}$ of an aqueous polymer solution was added dropwise to an equal volume of BSA NPs suspension under constant shaking of $500 \mathrm{rpm}$. Shaking was continued for $1 \mathrm{hr}$.

To obtain encapsulation efficiency, NPs containing FAMlabeled siRNA were centrifuged at $15000 \mathrm{rpm}$ for 30 minutes. The FAM-labeled siRNA in the supernatant and pellet were determined using a plate reader $\left(\lambda_{\mathrm{EX}}: 485 \mathrm{~nm}, \lambda_{\mathrm{EM}}\right.$ : $527 \mathrm{~nm}$ ) and a calibration curve based on known concentrations of FAM-labeled siRNA. The encapsulation efficiency was calculated as (FAM-siRNA pellet/(FAM-siRNA pellet + FAM-siRNA supernatant $)) \times 100 \%$. FAM-labeled siRNA was also used to study the apparent release kinetics of siRNA from BSA NPs coated with different polymers. Polymer concentration of $0.3 \mathrm{mg} / \mathrm{mL}$ was used to coat BSA NPs and release values were normalized to $0 \%$ for day 0 , as described previously [13]. The suspensions were incubated at $37^{\circ} \mathrm{C}$ in PBS under shaking and aliquots were taken at predetermined time points and centrifuged at $15000 \mathrm{rpm}$ for 30 minutes. The siRNA in the supernatant and pellet were determined using a calibration curve.

2.7. Cell Uptake Studies. To assess cellular uptake of NPs, human breast cancer MDA-231 cells were used. Two sets of NPs were prepared for cell uptake; (i) uncoated and coated BSA NPs with no siRNA, and (ii) uncoated and coated BSA NPs loaded with FAM-labeled siRNA. The siRNA encapsulation was achieved as described above, except that $20 \mu \mathrm{L}$ of siRNA solution $(0.15 \mathrm{mg} / \mathrm{mL})$ was used for FAMlabeled siRNA encapsulation. $1000 \mu \mathrm{L}$ of aqueous polymer solution was added to $2000 \mu \mathrm{L}$ of BSA NPs suspension (final concentration: $0.3 \mathrm{mg} / \mathrm{mL}$ ) under shaking for $1 \mathrm{hr}$ to achieve coating. The suspensions were then dialyzed against DMEM for 24 hrs with two changes in dialysis solution.

For uptake, a monolayer of MDA-MB-231 cells were seeded in 24-well plates and allowed to attach for $24 \mathrm{hrs}$ 
TABLE 1: Selected characteristics of prepared NPs: (i) efficiency of PLL and PEG-PLL coating on BSA NPs, (ii) $\zeta$-potential, and (iii) average size (average \pm standard deviation, $n \geq 5$ ). Note that the NPs from $4.2 \mathrm{kDa}$ PLL-PEG coating gave two size populations with relative ratios of $14 \%$ and $86 \%$.

\begin{tabular}{lccr}
\hline & Coated amount $(\mathrm{mg}$ conjugate/mg BSA $)$ & $\zeta$-Potential $(\mathrm{mV})$ & Diameter $(\mathrm{nm})$ \\
\hline Uncoated & - & $-10.1 \pm 0.4$ & $255 \pm 30$ \\
$4.2 \mathrm{kDa}$ PLL & $0.12 \pm 0.03$ & $10.1 \pm 0.5$ & $350 \pm 90$ \\
$24 \mathrm{kDa}$ PLL & $0.10 \pm 0.02$ & $20.4 \pm 0.5$ & $310 \pm 30$ \\
$4.2 \mathrm{kDa}$ PEG-PLL & $0.016 \pm 0.006$ & $1.8 \pm 0.3$ & $220 \pm 26(14 \%)$ \\
$24 \mathrm{kDa}$ PEG-PLL & $0.007 \pm 0.003$ & $7.8 \pm 0.9$ & $880 \pm 100(86 \%)$ \\
\hline
\end{tabular}

to reach $\sim 50 \%$ confluency (see [16] for culture conditions on the cells). The medium was replaced with $500 \mu \mathrm{L}$ of fresh DMEM with $10 \%$ FBS and $1 \%$ antibiotics (penicillin/streptomycin). Then, $500 \mu \mathrm{L}$ of NP suspension in DMEM was added to the cells (in triplicate) and the cells were incubated for $24 \mathrm{hrs}$ at $37^{\circ} \mathrm{C}$ in a humidified atmosphere of $95 \%$ air $/ 5 \% \mathrm{CO}_{2}$. After the incubation period, cells were washed with HBSS $(\times 2)$ and trypsinized. A 3.7\% formaldehyde solution was added to suspended cells and the siRNA uptake was quantified by a Beckman Coulter QUANTA SC flow cytometer using the FL1 channel to detect cell-associated fluorescence. The percentage of cells showing FAM-fluorescence and the mean fluorescence in total cell population were determined. Calibration was performed by gating with the negative control (i.e., "No Treatment") group such that the autofluorescent cell population represented 1$2 \%$ of the total cell population.

\section{Results and Discussion}

The nature of the NP coating, in addition to its role in NP stabilization, is expected to control the apparent release of the encapsulated therapeutic agents whilst creating an interface that inhibits nonspecific protein adsorption. In addition to this, it is desirable to evaluate if PEGylation of the PLL based NP coating affects cytotoxicity and/or the cellular uptake of siRNA. Towards this end, NPs were coated with 4.2 and $24 \mathrm{kDa}$ PLLs and PEG conjugates of the same. The use of PLL was considered advantageous as compared to previously employed PEI since the latter is synthetic, highly cytotoxic to mammalian cells, and undergoes an ill-defined degradation pattern. Unlike chemical crosslinkers, such as glutaraldehyde, the coating approach employed is thought to be a more bioacceptable means to stabilize the particles and further provides a convenient means of surface control. After characterization of the NP features, protein adsorption, composition of adsorbed protein layer, apparent siRNA release, cytotoxicity, and cellular uptake of siRNA were assessed.

3.1. PEG-PLL Conjugation. Conjugation of $\mathrm{mPEG}-\mathrm{NHS}$ to PLL was demonstrated through the addition of varying quantities of PEG and subsequent NMR analysis after dialysis to determine the resulting PEG:PLL molar ratio. The maximum conjugation ratio of PEG : PLL was determined to be $\sim 26$ and 150 for 4.2 and $24 \mathrm{kDa}$ PLL systems, respectively (results not shown). On average, this translated into the incorporation of $\sim 6$ PEG per $1 \mathrm{kDa}$ of PLL for both 4.2 and $24 \mathrm{kDa}$ systems. However, given that the PDI of the PLL and PEG polymer was not one, these values are considered on average.

3.2. NP Characterization. The amount of PLL or PEG-PLL incorporated into the coating used to stabilize the NPs was determined so as to evaluate the effect of the presence of PEG upon $\zeta$-potential and resulting NP size. It was evident that unmodified PLLs, regardless of size, incorporated similar mass amounts into the NP coating layer of $\sim 0.1 \mathrm{mg}$ PLL per mg BSA (Table 1). Although, it is evident that on a mole basis there would be more molecules of $4.2 \mathrm{kDa}$ PLL adsorbed than $24 \mathrm{kDa}$ systems, PLL adsorption is largely driven by electrostatic forces so the amount of PLL needed to occupy the charges on the surface of the NP was similar, regardless of individual chain lengths. Moreover, this amount of PLL in the coating layer agreed with previously published results for similar systems [13]. However, PEGylated 4.2 and $24 \mathrm{kDa}$ PLL systems showed an adsorbed amount of PEG-PLL at $16 \pm 6$ and $7 \pm 3 \mu$ g conjugate per mg BSA, respectively. An order of magnitude decrease in adsorbed mass was seen in the stabilizing layer upon PLL PEGylation. It is likely that this significant decrease in adsorbed mass was due to steric hindrances imposed by preadsorbed PEG conjugates that prevented other PEG-PLLs from reaching the interface or from the screening of PLL charges that would reduce the driving force for PEG-PLL incorporation into the film. Differences observed between 4.2 and $24 \mathrm{kDa}$ PEGPLL coatings suggest that the smaller polymer conjugate may better fill the surface of the NP.

Mean particle sizes and $\zeta$-potentials for PLL-coated NPs were similar to previously reported values (Table 1) [13]. NPs formed with 4.2 and $24 \mathrm{kDa}$ PLLs yielded statistically similar average particle sizes of $\sim 350$ and $310 \mathrm{~nm}$, respectively; results similar to previously reported values for PLL coated BSA NPs [13]. Upon using PEG-PLL for stabilizing the BSA NPs, the diameter of the NPs increased dramatically. For $4.2 \mathrm{kDa}$ PEG-PLL systems a bimodal distribution in particle diameter was observed, where $\sim 14$ and $86 \%$ of the NP population had diameters of $\sim 220$ and $880 \mathrm{~nm}$, respectively. Systems composed of $24 \mathrm{kDa}$ PEG-PLL systems had an average diameter of $\sim 845 \mathrm{~nm}$. While the difference between systems using different PLL MWs was negligible, it is obvious that the differences observed upon incorporation of 
TABLE 2: BSA NP plasma adsorption conditions and results as evaluated with immunoblots.

\begin{tabular}{|c|c|c|c|c|c|c|}
\hline & \multirow{2}{*}{ Fragment size $(\mathrm{kDa})$} & \multirow{2}{*}{ Fragment name } & \multicolumn{4}{|c|}{ System } \\
\hline & & & 4.2 PLL & 24 PLL & 4.2 PEG-PLL & 24 PEG-PLL \\
\hline \multirow{4}{*}{ Fibrinogen } & 48 & $\gamma$ & $* * *$ & $* *$ & $* *$ & $* * *$ \\
\hline & 56 & $\beta$ & $* * *$ & $* *$ & $* * *$ & $* * *$ \\
\hline & 68 & $\alpha$ & $* * *$ & $* * *$ & $* * *$ & $* * *$ \\
\hline & $<48$ & Cleavage & $*$ & 0 & $*$ & $* *$ \\
\hline Albumin & 66 & & $* * *$ & $* * *$ & $* * *$ & $* * *$ \\
\hline \multirow{3}{*}{ C3 } & 42 & Activation & $* *$ & $*$ & $* *$ & $* *$ \\
\hline & 70 & $\beta$ & $*$ & $* *$ & $* *$ & $* *$ \\
\hline & 115 & $\alpha$ & 0 & 0 & 0 & 0 \\
\hline Apolipoprotein A-1 & 27 & & $0+$ & $0+$ & 0 & 0 \\
\hline \multirow{2}{*}{ Plasminogen } & 25 & & 0 & 0 & 0 & 0 \\
\hline & 60 & & $0+$ & $*$ & 0 & 0 \\
\hline
\end{tabular}

Plasma proteins eluted with $1.0 \mathrm{~mL}$ of $2 \%$ SDS in PBS; 0 indicates zero band intensity, while $0+$ indicates trace band intensity and $* * *$ indicates highest intensity bands. Proteins shown in Table 1 but absent from this table were not observed in immunoblotting (zero band intensity throughout).

PEG were not. This large difference may be a direct result of the steric hindrances imposed by adsorbed PEG-PLL leading to lower amount of conjugate being incorporated into the stabilizing coating. With less conjugated PEG-PLL filling the surface, a larger NP may form.

The $\zeta$-potentials of all four types of NPs were positive, suggesting sufficient PLL or PEG-PLL adsorbed to offset the inherent negative $\zeta$-potential of the BSA NP. The $\zeta$ potential for the 4.2 and $24 \mathrm{kDa}$ PLL systems were $\sim 10$ and $20 \mathrm{mV}$, respectively. The $\zeta$-potentials for similar systems were found to plateau, with respect to increasing PLL concentration, around these values, suggesting that the NP surfaces were nearly saturated [13]. The PEG-PLL coating instead appeared to reduce the $\zeta$-potential, as the 4.2 and $24 \mathrm{kDa}$ PEG-PLL constructs had $\zeta$-potentials of $\sim 1.8$ and $7.8 \mathrm{mV}$, respectively. Low average $\zeta$-potentials for the PEGPLL coated NPs seemed to suggest the presence of PEG, as PEG should result in a less charged surface as well as possibly screening $\zeta$-potentials. It is interesting that there was a higher $\zeta$-potential for the 24 versus $4.2 \mathrm{kDa}$ PEG-PLL system given that the $4.2 \mathrm{kDa}$ system adsorbed more PEGPLL material. It may be that the more $4.2 \mathrm{kDa}$ PEG-PLL molecules result in a more compressed PEG layer that shields the $\zeta$-potentials of the PLL and thus lower the $\zeta$-potential. Whereas the $24 \mathrm{kDa}$ PEG-PLL film has more flexible PEG chains (i.e., mushroom regime) that may allow for more of the $\zeta$-potential to be measured. The literature has shown that PEI-PEG systems observed a $\zeta$-potential plateau at $\sim 14 \mathrm{mV}$, which was greater than that observed herein [12]. A weak positive charge $(\zeta$-potential $<+5 \mathrm{mV})$ has been suggested for minimally adsorbing surfaces $[17,18]$ and PEG-coated NPs prepared in this study fulfill this feature, and surface PEGs could further improve the stability for such low $\zeta$-potential NPs. Moreover, this low $\zeta$-potential may also mediate NP aggregation; however, previous studies have shown that NP sizes are highly dependent on coating properties [13].

3.3. Protein Adsorption to NP Systems. The adsorption of proteins at the NP-blood interface is crucial to several important aspects of drug delivery, where a decrease in the amount of adsorbed protein may lead to an increase in the effectiveness of incorporating tethered targeting molecules on the NP surface. Moreover, decreasing protein adsorption may lead to increased circulation times by decreasing opsonization and potential host responses to the NP. Thus, in order to understand how the differences in the coating affects both nonspecific protein adsorption as well as the composition of the adsorbed layer, 4.2 and $24 \mathrm{kDa}$ PLL and PEGylated versions of these PLLs were evaluated using platelet poor plasma adsorption, where adsorbed proteins were eluted from the surface using a $2 \%$ SDS incubation. As there is no way to accurately control the total surface area of NPs in solution, or to accurately estimate it, conducting a total protein analysis would not be indicative of the amount of adsorbed protein per surface area. Moreover, as it has been shown previously that 4.2 and $24 \mathrm{kDa}$ PLL systems do not leak more than $1 \%$ of the BSA incorporated into the formed NPs within $2 \mathrm{hrs}$, all eluted proteins are most likely from adsorbed protein [13].

Since immunoblot analysis is qualitative for detecting protein levels, and more informative for determining protein presence, equal volumes of eluted protein solution were loaded $(50 \mu \mathrm{L})$, being a commonly employed technique. The results of the immunoblot analysis for the adsorbed plasma proteins on NPs are summarized in Table 2. The presence of high levels of fibrinogen and fibrinogen fragments suggests active coagulation in all samples except for the $24 \mathrm{kDa}$ PLL NPs. It is possible that fibrinogen might have been less easily eluted from these surfaces. Adsorbed fibrinogen has previously been shown to activate platelets and induce the accumulation of phagocytes [19, 20].

High intensity bands for human serum BSA were observed for all formulations. BSA adsorption in these systems is not unusual as the surfaces were formed with polymers that bind avidly to BSA. BSA is an unreactive protein that displays anticell adhesion and provides "passivation" properties, so that the presence of BSA in such great quantities on all of 
the NP systems is promising from a biocompatibility standpoint $[21,22]$.

Complement activation is a response against foreign surfaces with important implications for biocompatibility of administered agents [23]. Complement activation pathways are triggered by a variety of stimuli but ultimately serve to cause opsonization through the activation of C3 [24]. C3 is composed of $\alpha(115 \mathrm{kDa})$ and $\beta(70 \mathrm{kDa})$ peptide chains. If complement is activated and the C3 cleaved, a $42-\mathrm{kDa}$ fragment is created. The $42 \mathrm{kDa}$ C3 fragment and the 70 $\mathrm{kDa} \beta$-fragment were both present in relatively significant quantities on all NP systems. The presence of the $42-\mathrm{kDa}$ C3 fragment indicated complement activation. PLL-coated NPs appeared to adsorb less $70 \mathrm{kDa}$ C3. While this may be the case, it is important to consider that $70 \mathrm{kDa}$ C3 may simply be less readily eluted from these surfaces than the others. The $115-\mathrm{kDa} \alpha \mathrm{C} 3$ fragment was not observed on any of the systems. It is possible that the NPs did generate this fragment, but it did not adsorb to the NP surfaces.

Trace levels of apolipoprotein A-1 were found on both PLL-coated NP systems, while none was observed on PEGPLL coated NPs. The presence of apolipoprotein A-1 implies anti-inflammatory activity. The literature states that HDLs such as apolipoprotein A-1 are also capable of endothelial protection, including the control of cell proliferation, the inhibition of apoptosis, the modulation of the secretory functions, the regulation of coagulation, fibrinolysis, and platelet adhesion, and the inhibition of inflammatory processes. Similar to the apolipoprotein A-1 case, $60 \mathrm{kDa}$ plasminogen was found in moderate quantities on the $24 \mathrm{kDa}$ PLL system, with minor amounts in $4.2 \mathrm{kDa}$ PLL coated NPs and none on PEG-PLL coated NPs. This data suggests that PEG can effectively block plasminogen adsorption in these NP systems. Plasminogen binding is likely to be facilitated due to the epsilon amines of the PLL chains [25]. The presence of surface-localized plasminogen may actually enhance the biocompatibility characteristics of the NPs, as it is the precursor for plasmin, which has the potential for clot dissolution [25]. Further studies are needed to discern if the surface-adsorbed plasminogen indeed can be converted to plasmin within blood while localized to the NP surface.

In addition to these five proteins, sixteen other proteins were screened without detection, even in trace amounts, for any of the NP formulations. These fifteen include high molecular weight kininogen (HMWK), low molecular weight kininogen (LMWK), factor I, fibronectin, $\alpha_{1-}$ antitrypsin, thrombin, prothrombin, protein $\mathrm{C}$, vitronectin, protein $\mathrm{S}$, prekallikrein, antithrombin, immunoglobulin $\mathrm{G}$ (IgG), factor XII, factor XI, and $\alpha_{2}$-macroglobulin. The lack of the contact phase coagulation proteins of the intrinsic clotting cascade, prekallikrein, HMWK, factor XI and factor XII (Table 2) implied that the NPs should not be procoagulant. HMWK has been shown to both enhance biocompatibility through its anticell adhesion properties, as well as to hinder it by acting as a cofactor for the contact phase of coagulation; therefore, it is unclear whether the presence of this protein on the surfaces is desirable [26, 27]. Further along the cascade, the absence of prothrombin and thrombin reinforces the inference that the systems are noncoagulant [28]. Fibrinogen was detected in significant quantities (Table 2), so the absence of thrombin is especially important to prevent fibrin formation.

The anticoagulation pathway was also monitored via the immunoblots. Protein C, a significant component of anticoagulation, was not observed in significant quantities. Protein S, a cofactor for Protein C, and vitronectin, an indirect inhibitor of plasminogen conversion to plasmin, were also not detected, indicating that the proteins controlling anticoagulation were not present. Two proteins involved in both coagulation and anticoagulation pathways, $\alpha_{2}$-macroglobulin and antithrombin, were investigated, but not detected again. Antithrombin is an uncharged serine protease inhibitor that is responsible for limiting irregular clotting [28]. Due to the absence of thrombin, the absence of $\alpha_{2}$-macroglobulin is inconsequential for the coagulation pathway. $\alpha_{2}$-Macroglobulin inhibits plasmin in the anticoagulation pathway, but was not detected. Antithrombin, which has a variety of targets in both the coagulation and anticoagulation pathways, was not observed in any of the NP systems.

The absence of other proteins not involved in clotting or fibrinolysis cascades is informative. Lack of IgG adsorption suggests a lack of reactivity by the circulating antibodies and no subsequent stimulation of the immune response. The lack of $\operatorname{IgG}$ also indicates that the possible complement activation seen (based on the presence of C3 fragments; Table 2) occurs via the alternative pathway only. The $\alpha_{1}$-antitrypsin is an important serine proteases in the body [29]. $\alpha_{1}$-Antitrypsin has a charge of -12 at a $\mathrm{pH}$ of 7.0, so its adsorption to the positively charged NPs would be expected. However, it was not detected in the immunoblot analysis. It is possible that other negatively charged proteins are preferentially adsorbed to the surface of the NPs, neutralizing its charge.

3.4. siRNA Encapsulation Efficiency and Release. siRNA encapsulation as a function of coating conditions was explored (Figure 1). Respective encapsulation efficiencies ranged from $16 \pm 2 \%$ to $53 \pm 7 \%$ for uncoated and $4.2 \mathrm{kDa}$ PEG-PLL coated NPs. Statistically significant differences in encapsulation efficiency from uncoated NPs were observed for NPs with $4.2 \mathrm{kDa}$ PLL $(P<0.005), 4.2 \mathrm{kDa}$ PEG-PLL $(P<0.05)$, and $24 \mathrm{kDa}$ PEG-PLL $(P<0.05)$. In almost all cases, the use of a coating increased the encapsulation efficiency. Previous studies have shown that when PEI was used to stabilize poly(D,L-lactide-co-glycolide) NPs, the encapsulation efficiency increased from $\sim 43-80 \%$ [30]. The cationic polymers presumably sequester the siRNA from freely diffusing during the fabrication process and help to retain the therapeutic agent within the NPs. Using PEG-substituted PLL for coating resulted in increased encapsulation efficiency for both 4.2 and $24 \mathrm{kDa}$ PLL systems. Furthermore, the $4.2 \mathrm{kDa}$ PLL systems, with or without PEG, exhibited nearly double the encapsulation efficiencies of their corresponding $24 \mathrm{kDa}$ PLL systems (Figure 1). Excluding the $24 \mathrm{kDa}$ PLL systems (which did not give statistical significance from uncoated NPs), these results demonstrate that encapsulation efficiency can be controlled through varying PLL size and the incorporation of PEGylated PLL moieties. 


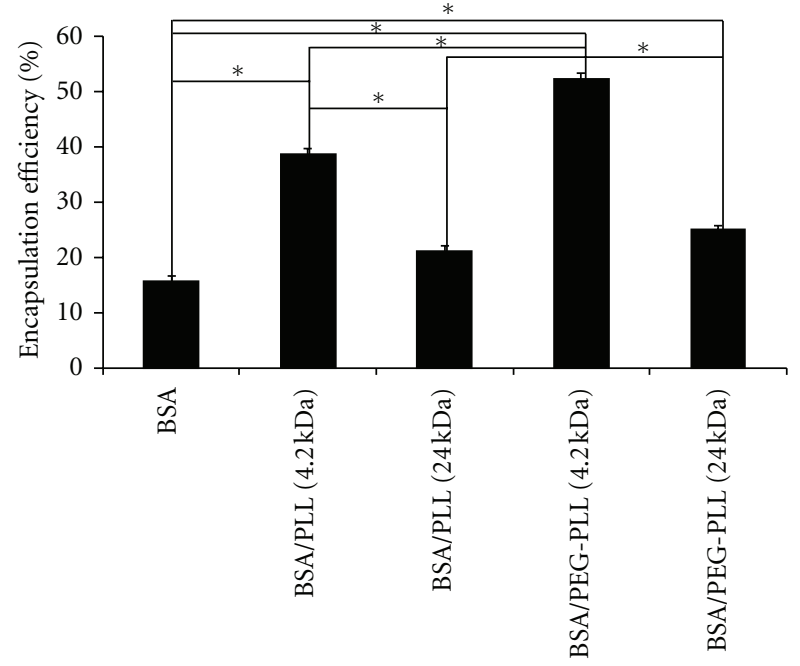

Figure 1: Encapsulation efficiencies of siRNA in various NPs. The value in parentheses represents the molecular weight of the PLL in $\mathrm{kDa}$. For statistical comparison via double-sided $t$-tests, one asterisk ${ }^{*}$ ) represents $P<0.05$ and data represent average $\pm 1 \mathrm{SD}, n>5$.

The siRNA release from the NPs coated with PLL and PEG-PLL conjugates was monitored over 7 days (Figure 2). It should be noticed that all systems studied had a minimal burst effect, which may suggest the incorporation of the siRNA within the NPs studied. The highest release was observed in the $4.2 \mathrm{kDa}$ PLL coated NPs, which had a Day 7 release of $93 \pm 1 \%$, while the lowest release was observed in the $24 \mathrm{kDa}$ PLL coated NPs, with a Day 7 release of $33 \pm 1 \%$. Despite the similar adsorbed mass incorporated into the stabilizing layer for both 4.2 and $24 \mathrm{kDa}$ PLLs, the release profile of siRNA was drastically different. This may be an indication that the $24 \mathrm{kDa}$ PLL coatings form a more stable $\mathrm{NP}$ which may impede both the breakup of the NP and/or the diffusive release of siRNA. However, previous work in our lab has shown that 4.2 and $24 \mathrm{kDa}$ PLL stabilized NPs yield similar stabilities at the $0.3 \mathrm{mg} / \mathrm{mL}$ condition [13]; thus, is it likely that the differences observed are most likely due to an increase in resistance to diffusive forces leading to a slower release profile for the $24 \mathrm{kDa}$ PLL systems.

Interestingly, the effect of incorporating PEG into the NP coating had a different effect upon siRNA release for 4.2 and $24 \mathrm{kDa}$ PLL systems. After 7 days it was observed that $4.2 \mathrm{kDa}$ coated NPs showed a decrease in siRNA release from $93 \pm 1 \%$ to $62 \pm 25 \%(P<0.05)$ upon incorporating PEG, whereas for $24 \mathrm{kDa}$ an increased release from $33 \pm 1$ to $43 \pm 12 \%$ occurred upon PEG presence; the latter trend was not statistically significant. These data may coincide with the discussion regarding the density of the PEG-PLL layers highlighted by the $\zeta$-potential studies. Namely, that the $4.2 \mathrm{kDa}$ PLL layer adsorbed more PEG-PLL than the $24 \mathrm{kDa}$ system, yet had a lower net charge that may suggest a denser PEG layer that shielded some of the $4.2 \mathrm{kDa}$ PLL charge. Thus, it is probable that the large $24 \mathrm{kDa}$ PEG-PLL conjugate was not as able to fill the surface an impede passive siRNA release as compared to the $4.2 \mathrm{kDa}$ conjugate. Taken together,

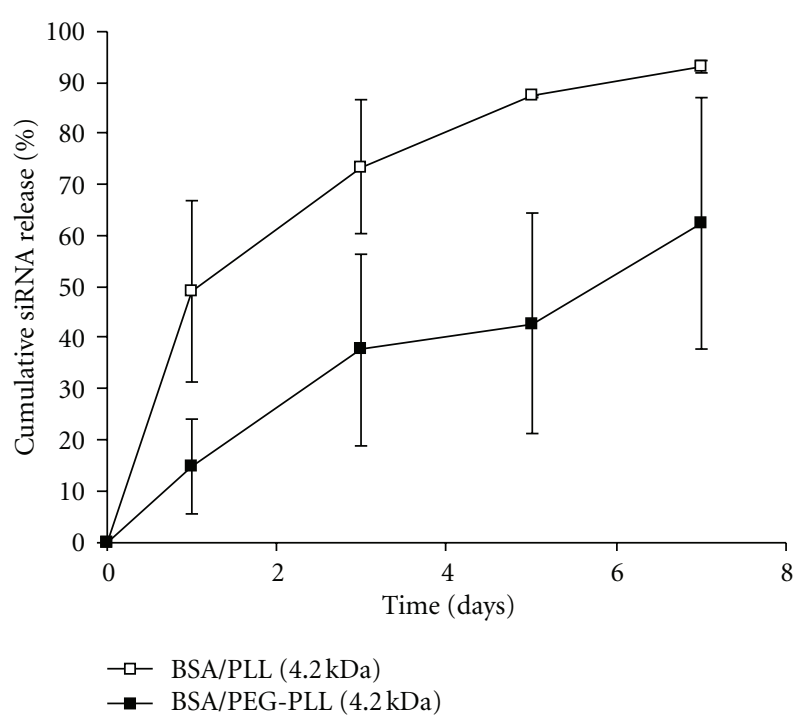

(a)

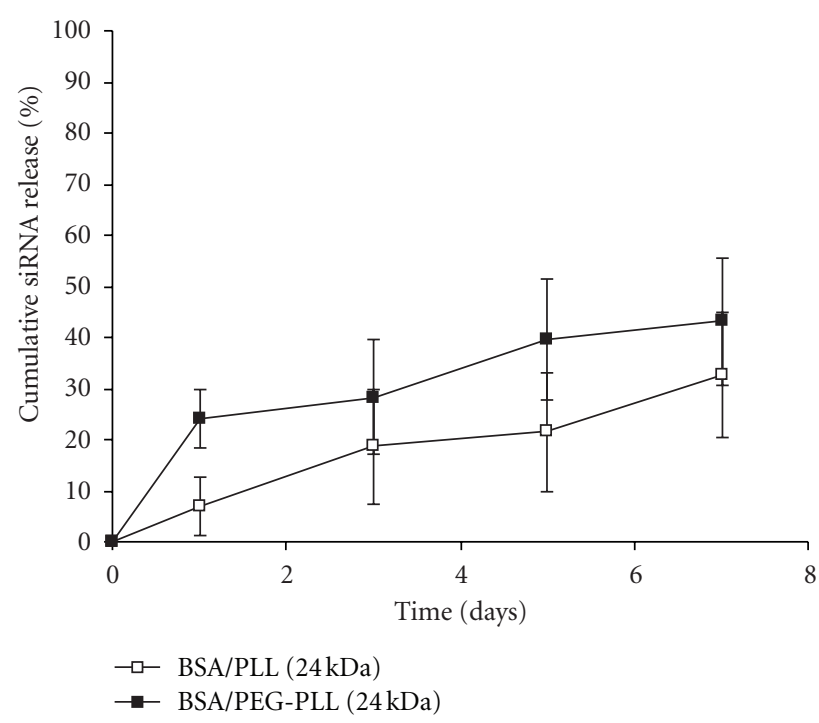

(b)

Figure 2: Cumulative siRNA release profile for $4.2 \mathrm{kDa}$ (a) and $24 \mathrm{kDa}$ (b) PLL-based coatings, over seven days. Trend lines are provided as a guide to the eye only. Data points represent an average $\pm 1 \mathrm{SD}, n \geq 3$.

these results indicated that, based upon the presence/absence of PEG and the size of PLL, siRNA release from BSA NPs can be controlled over a range of $\sim 20 \%$ to $90 \%$ over 7 days. Previously, mPEG-PGLA-PLL coated NPs [31] yielded a similar release profile, including $\sim 85 \%$ release after 7 days. Related in vivo work using solid lipid NPs [32] had similar release profiles except for the fact that they observed an initial burst release of $\sim 20 \%$ that was not observed herein. Through modifying NP creation parameters in this lipid study, the overall release could be varied from $\sim 70-90 \%$ over a period of 7 days. An experiment involving PLGA-PLL NPs (with adsorbed PEG to improve circulation time) found a plateau in the release profile at $\sim 55 \%$ after 7 days [33]. 


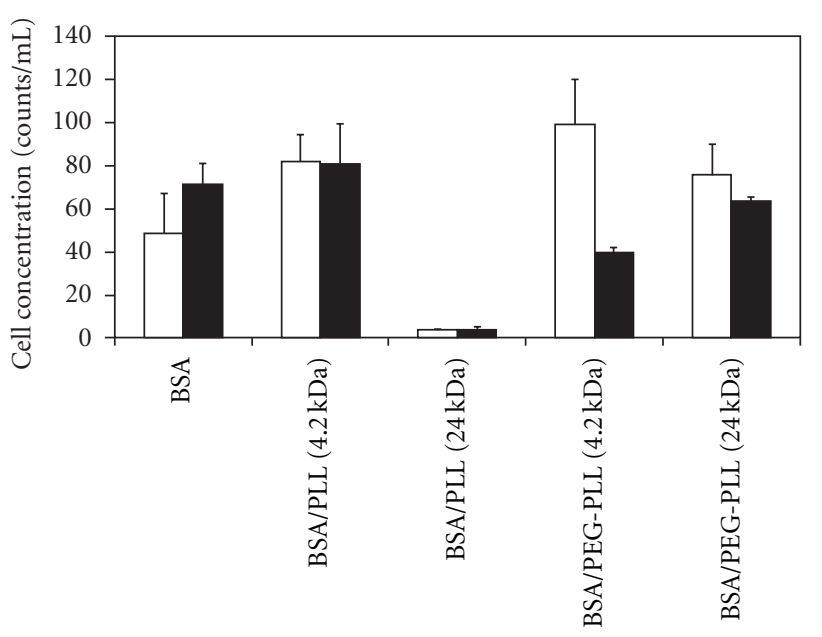

Figure 3: Cell concentrations after exposure to blank NPs ( $\square$ ) and FAM-siRNA-containing NPs $(\boldsymbol{\square})$. The value in parentheses represents the molecular weight of the PLL in $\mathrm{kDa}$. The NPs coated in PEG-PLL ( $4 \mathrm{kDa}$ ) and containing FAM-siRNA showed the greatest cell concentrations. Data represent average $\pm 1 \mathrm{SD}, n>5$.

3.5. Cellular Uptake. siRNA uptake was investigated in order to ascertain the delivery potential of the NPs as a function of coating properties. Flow cytometry was used to detect siRNA uptake based on NPs containing FAM-labeled siRNA, as well as the cell counts from the cultures exposed to the NPs. The latter is representative of cell survival upon incubation with the NPs containing no siRNA or FAM-labeled siRNA (Figure 3). Uncoated NPs and NPs coated with $4.2 \mathrm{kDa}$ PLL had similar cell counts, irrespective of the presence or absence of encapsulated siRNA. Coating with $24 \mathrm{kDa}$ PLL caused a dramatic drop in cell numbers, clearly indicating the toxicity of this type of coating irrespective of the presence or absence of encapsulated siRNA (Figure 3). After coating with PEG-substituted PLLs, there was little toxicity for the $24 \mathrm{kDa}$ PLL for blank NPs and siRNA-containing NPs $>20$-fold increase in toxicity. With $4 \mathrm{kDa}$ PLL, using PEG-substituted PLL gave better cells counts in the absence of siRNA but somehow reduced cell counts in the presence of siRNA $(P<$ 0.05 between the two groups). No other system showed such a difference with and without siRNA (based on paired $t$ test). The encapsulated siRNA was nonspecific and was not expected on its own to cause cell toxicity. It is possible that it might have resulted in nonspecific effects once inside the cells since the molecule is highly charged and it might interact with cationic molecules critical for cell survival (such as histones, etc.), ultimately disrupting the normal cellular physiology. This issue needs to be further explored in future studies.

The siRNA uptake is summarized in Figure 4 as the mean uptake (Figure 4(a)) or the percentage of cells positive for siRNA (Figure 4(b)). The mean fluorescence of the cells exposed to NPs without FAM-siRNA was not statistically different among the NPs (as expected), and represented the background readings (i.e., normal autofluorescence). Compared to uncoated NPs, cells exposed to coated NPs

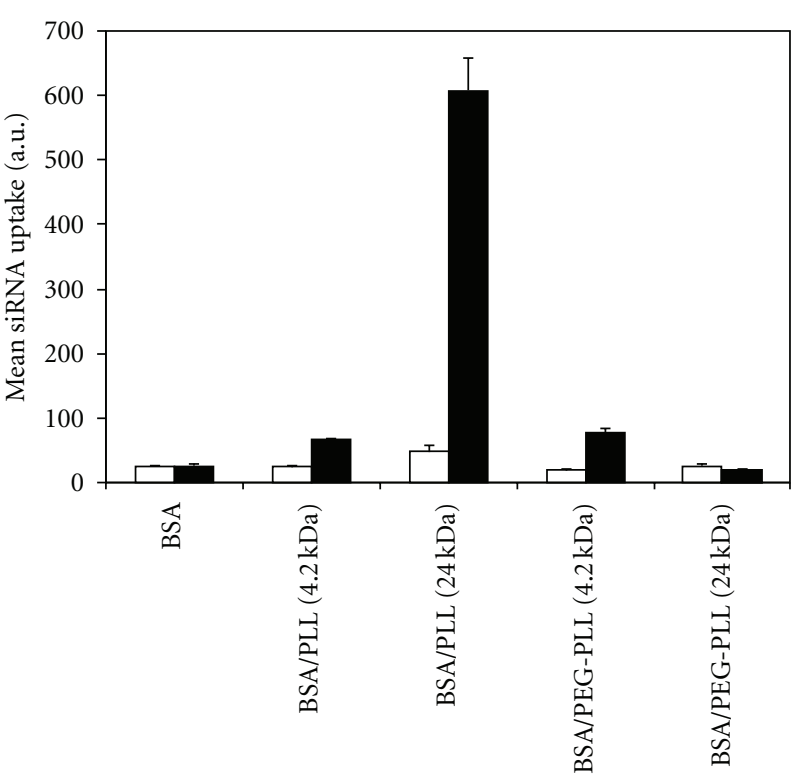

(a)

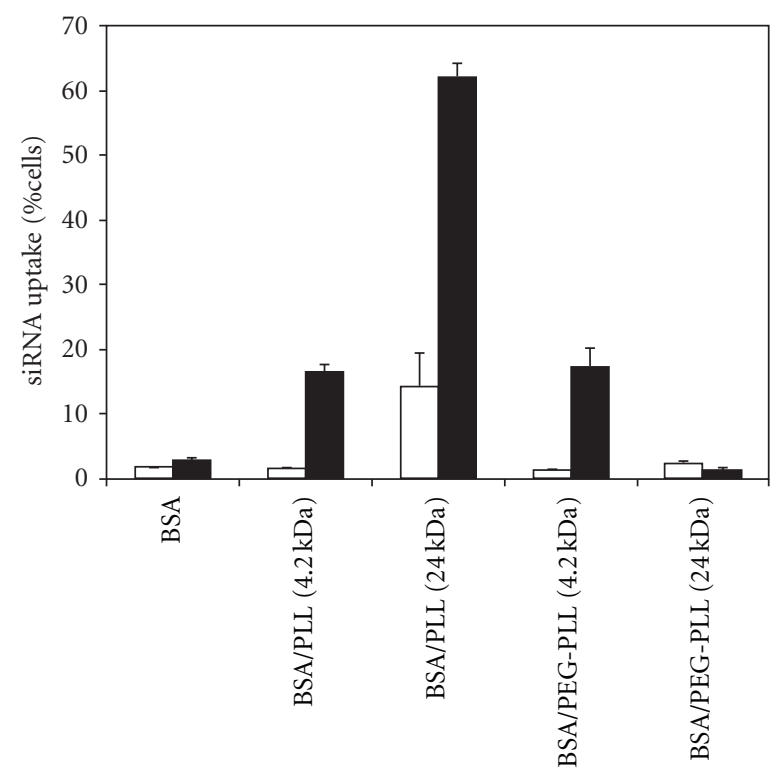

(b)

FIGURE 4: Data summarizing the mean uptake and percent cellular uptake of labeled and unlabeled siRNA. (a) Mean (+1 SD) FAM fluorescence of the cells exposed to NPs without siRNA ( $\square$ ) and with siRNA ( $)$. BSA NPs coated with $24 \mathrm{kDa}$ PLL showed the greatest cellular uptake. (b) Mean (+1 SD) siRNA-positive cells when the cells were exposed to NPs without siRNA ( $\square$ ) and with siRNA $(\square)$. BSA NPs coated with $24 \mathrm{kDa}$ PLL showed the greatest value of siRNA-positive cell population. The value in parentheses represents the molecular weight of the PLL in $\mathrm{kDa}$.

containing FAM-siRNA all had greater fluorescence than the background $(P<0.01$ for NPs coated with $4 \mathrm{kDa}$ PLL, $24 \mathrm{kDa}$ PLL and $4 \mathrm{kDa}$ PEG-PLL), except the NPs coated with $24 \mathrm{kDa}$ PEG-PLL. The latter did not show any evidence of increased uptake based on mean fluorescence of the cells. Although it is unknown why modification of the $24 \mathrm{kDa}$ PLL NPs with PEG 
resulted in an insignificant amount of uptake (compared to controls), it is possible that the large average diameter of $\sim 800 \mathrm{~nm}$ may prohibit cellular uptake. It was clear that the NPs coated with $24 \mathrm{kDa}$ PLL had the highest cellular delivery of FAM-labeled siRNA. This was consistent with toxicity results that indicated highest toxicity (i.e., cell interaction) with this type of NPs. While the presence of PEG did not affect uptake with $4.2 \mathrm{kDa}$ PLL, an apparent dramatic effect of PEG was evident with the $24 \mathrm{kDa}$ PLL. The proteinrepellent properties of PEG presumably prevented binding to cell surfaces, which is critical for internalization and siRNA uptake. This result was also in line with toxicity results, where the cells displayed much more tolerance to $24 \mathrm{kDa}$ PEG-PLL coated NPs.

Figure 4(b) summarizes that uptake of BSA NPs among the cell population exposed to the NPs. Note that the uptake was minimal in the absence of coating (i.e., pure BSA NPs) and for NPs containing no FAM-labeled siRNA, which served as the background control (1-3\% siRNA-positive cell population). The only exception to this observation was the NPs coated with the $24 \mathrm{kDa}$ PLL; a high percentage of cells (14.4\%) became auto-fluorescent that yielded significantly high proportion of "apparently" siRNA-positive cells. We previously made such an observation when NPs imparted certain toxicity on the cells [34]. For example, when cells are exposed to blank NPs with no reporter genes such as GFP, they display GFP-like fluorescence (with similar excitation/emission characteristics to FAM) even in the absence of a reporter gene. It is not surprising that the most toxic formulation in this study behaved in this way as well.

With NPs coated by $4.2 \mathrm{kDa}$ PLL and $4.2 \mathrm{kDa}$ PEGPLL, $\sim 16$ and $17 \%$ of the cells, respectively, yielded siRNApositive cells, clearly indicating the beneficial effect of this coating on the cellular delivery of the NPs. With NPs coated by $24 \mathrm{kDa}$ PLL, $62.2 \%$ of the cells yielded siRNA-positive cells, but using the same MW PLL with PEG substitution abolished the uptake totals (note the lack of difference in cell uptake for between siRNA-positive and siRNA-negative NPs). Considering the auto-fluorescence obtained in the cells exposed the $24 \mathrm{kDa}$ PLL coated NPs, we expect the uptake to be closer to $\sim 48 \%$ in this case. PEG obviously plays a significant role in this case, preventing the uptake of the NPs. This is in line with previous protein adsorption results, which indicated relatively less binding of plasma proteins to the NPs. It must be noted that the uptake values reported among the cell population should be considered as a relative measure to compare different NP formulations and not taken at absolute values. It is possible to significantly alter the measured values depending on the siRNA loading in NPs; with higher fluorescing NPs, higher rates of uptake could be obtained for the same formulations.

\section{Conclusions}

The stabilizing coating used on BSA NPs was expected to have significant implications on the physical characteristics of the formed NPs, blood plasma interactions, siRNA encapsulation, and cellular uptake. It was observed that the use of PEG increased average NP size and polymer coating on the NPs. In addition, PEG coatings were found to decrease nonspecific protein adsorption from human plasma as well as decrease the cytotoxicity of certain NPs (i.e., ones coated with highly toxic $24 \mathrm{kDa}$ PLL). This result was likely not due to size, but rather attributed to inherently higher toxicity of the high MW PLL. Although an extensive array of adsorbed proteins was found on the NP surfaces, proteins for both passivating the NPs as well as activating the foreign body reactions were noted. The benefit of a PEGylated NPs was not clear in this respect and further evaluation (in vitro or in vivo) will be necessary to fully reveal the biocompatibility of the NPs. For the NPs coated with $4.2 \mathrm{kDa}$ PLL, PEG also increased the siRNA encapsulation efficiency, maintained a similar cellular uptake, and delayed siRNA release over a period of 7 days. These desirable results suggest that NP engineering could be possible by controlling PLL size and the use of PEG to prevent the removal from the bloodstream without hindering the efficiency of drug delivery. It is thought that the fundamental knowledge acquired in this study will further the design of coating strategies for controlling the formation and biological interactions of BSA NPs in circulation for the express purpose of delivering drugs via systemic administration.

\section{Abbreviations}

$\begin{array}{ll}\text { BSA: } & \text { Bovine serum BSA } \\ \text { FAM: } & \text { 5-Carboxyfluorescein } \\ \text { MW: } & \text { Molecular weight } \\ \text { NP: } & \text { Nanoparticle } \\ \text { PEG: } & \text { Poly(ethylene glycol) } \\ \text { PLL: } & \text { Poly-L-lysine } \\ \text { siRNA: } & \text { Short interfering RNA. }\end{array}$

\section{Authors' Contribution}

H. Yogasundaram and M. S. Bahniuk are equally contributed.

\section{Acknowledgments}

This work was supported by Discovery Grants provided to Hasan Uludağ and Larry David Unsworth by the Natural Sciences and Engineering Research Council of Canada (NSERC), and an operating grant provided to Larry David Unsworth by the National Research Council-National Institute of Nanotechnology (NRC-NINT). The authors thank Chemical and Materials Engineering Department (University of Alberta, Canada) for the infrastructure and personnel support.

\section{References}

[1] H. Harashima, K. Sakata, K. Funato, and H. Kiwada, "Enhanced hepatic uptake of liposomes through complement activation depending on the size of liposomes," Pharmaceutical Research, vol. 11, no. 3, pp. 402-406, 1994.

[2] C. Weber, J. Kreuter, and K. Langer, "Desolvation process and surface characteristics of HSA-nanoparticles," International Journal of Pharmaceutics, vol. 196, no. 2, pp. 197-200, 2000. 
[3] G. Wang and H. Uludag, "Recent developments in nanoparticle-based drug delivery and targeting systems with emphasis on protein-based nanoparticles," Expert Opinion on Drug Delivery, vol. 5, no. 5, pp. 499-515, 2008.

[4] L. D. Unsworth, H. Sheardown, and J. L. Brash, "Proteinresistant polyethylene oxide-grafted surfaces: chain densitydependent multiple mechanisms of action," Langmuir, vol. 24, no. 5, pp. 1924-1929, 2008.

[5] K. S. Soppimath, T. M. Aminabhavi, A. R. Kulkarni, and W. E. Rudzinski, "Biodegradable polymeric nanoparticles as drug delivery devices," Journal of Controlled Release, vol. 70, no. 1-2, pp. 1-20, 2001.

[6] J. Milton Harris and R. B. Chess, "Effect of pegylation on pharmaceuticals," Nature Reviews Drug Discovery, vol. 2, no. 3, pp. 214-221, 2003.

[7] D. E. Owens and N. A. Peppas, "Opsonization, biodistribution, and pharmacokinetics of polymeric nanoparticles," International Journal of Pharmaceutics, vol. 307, no. 1, pp. 93 102, 2006.

[8] P. Kingshott, H. Thissen, and H. J. Griesser, "Effects of cloudpoint grafting, chain length, and density of PEG layers on competitive adsorption of ocular proteins," Biomaterials, vol. 23, no. 9, pp. 2043-2056, 2002.

[9] L. D. Unsworth, H. Sheardown, and J. L. Brash, "Polyethylene oxide surfaces of variable chain density by chemisorption of PEO-thiol on gold: adsorption of proteins from plasma studied by radiolabelling and immunoblotting," Biomaterials, vol. 26, no. 30, pp. 5927-5933, 2005.

[10] L. D. Unsworth, H. Sheardown, and J. L. Brash, "Protein resistance of surfaces prepared by sorption of end-thiolated poly(ethylene glycol) to gold: effect of surface chain density," Langmuir, vol. 21, no. 3, pp. 1036-1041, 2005.

[11] M. Bonomini, B. Pavone, V. Sirolli et al., "Proteomics characterization of protein adsorption onto hemodialysis membranes," Journal of Proteome Research, vol. 5, no. 10, pp. 2666 2674, 2006.

[12] G. Wang, C. Kucharski, X. Y. Lin, and H. Uludağ, "Bisphosphonate-coated BSA nanoparticles lack bone targeting after systemic administration," Journal of Drug Targeting, vol. 18, no. 8, pp. 611-626, 2010.

[13] H. D. Singh, G. Wang, H. Uludağ, and L. D. Unsworth, "PolyL-lysine-coated albumin nanoparticles: stability, mechanism for increasing in vitro enzymatic resilience, and siRNA release characteristics," Acta Biomaterialia, vol. 6, no. 11, pp. 42774284, 2010.

[14] H. D. Singh, I. Bushnak, and L. D. Unsworth, "Engineered peptides with enzymatically cleavable domains for controlling the release of model protein drug from "soft" nanoparticles," Acta Biomaterialia, vol. 8, no. 2, pp. 636-645, 2012.

[15] S. Zhang, J. E. Wright, N. Ozber, and H. Uludağ, "The interaction of cationic polymers and their bisphosphonate derivatives with hydroxyapatite," Macromolecular Bioscience, vol. 7, no. 5, pp. 656-670, 2007.

[16] H. M. Aliabadi, B. Landry, P. Mahdipoor, and H. Uludağ, "Induction of apoptosis by survivin silencing through siRNA delivery in a human breast cancer cell line," Molecular Pharmaceutics, vol. 8, no. 5, pp. 1821-1830, 2011.

[17] N. Cao, D. Cheng, S. Zou, H. Ai, J. Gao, and X. Shuai, "The synergistic effect of hierarchical assemblies of siRNA and chemotherapeutic drugs co-delivered into hepatic cancer cells," Biomaterials, vol. 32, no. 8, pp. 2222-2232, 2011.

[18] A. J. Convertine, D. S. W. Benoit, C. L. Duvall, A. S. Hoffman, and P. S. Stayton, "Development of a novel endosomolytic diblock copolymer for siRNA delivery," Journal of Controlled Release, vol. 133, no. 3, pp. 221-229, 2009.

[19] T. M. Massa, M. L. Yang, J. Y. C. Ho, J. L. Brash, and J. P. Santerre, "Fibrinogen surface distribution correlates to platelet adhesion pattern on fluorinated surface-modified polyetherurethane," Biomaterials, vol. 26, no. 35, pp. 7367-7376, 2005.

[20] L. Tang and J. W. Eaton, "Natural responses to unnatural materials: a molecular mechanism for foreign body reactions," Molecular Medicine, vol. 5, no. 6, pp. 351-358, 1999.

[21] G. A. Skarja, R. L. Kinlough-Rathbone, D. W. Perry, F. Rubens, and J. L. Brash, "A cone-and-plate device for the investigation of platelet biomaterial interactions," Journal of Biomedical Materials Research, vol. 34, no. 4, pp. 427-438, 1997.

[22] S. Thakurta and A. Subramanian, "Evaluation of in situ albumin binding surfaces: a study of protein adsorption and platelet adhesion," Journal of Materials Science: Materials in Medicine, vol. 22, no. 1, pp. 137-149, 2011.

[23] L. Tang, L. Liu, and H. B. Elwing, "Complement activation and inflammation triggered by model biomaterial surfaces," Journal of Biomedical Materials Research, vol. 41, no. 2, pp. 333-340, 1998.

[24] H. Molina, "Complement and immunity," Rheumatic Disease Clinics of North America, vol. 30, no. 1, pp. 1-18, 2004.

[25] J. L. Brash, "Exploiting the current paradigm of bloodmaterial interactions for the rational design of blood-compatible materials," Journal of Biomaterials Science, Polymer Edition, vol. 11, no. 11, pp. 1135-1146, 2000.

[26] S. Asakura, R. W. Hurley, K. Skorstengaard, I. Ohkubo, and D. F. Mosher, "Inhibition of cell adhesion by high molecular weight kininogen," Journal of Cell Biology, vol. 116, no. 2, pp. 465-476, 1992.

[27] R. G. Flemming, R. A. Proctor, and S. L. Cooper, "Bacterial adhesion to functionalized polyurethanes," Journal of Biomaterials Science, Polymer Edition, vol. 10, no. 6, pp. 679-697, 1999.

[28] E. W. Davie and J. D. Kulman, "An overview of the structure and function of thrombin," Seminars in Thrombosis and Hemostasis, vol. 32, no. 1, pp. 3-15, 2006.

[29] P. G. W. Gettins, "Serpin structure, mechanism, and function," Chemical Reviews, vol. 102, no. 12, pp. 4751-4803, 2002.

[30] Y. Patil and J. Panyam, "Polymeric nanoparticles for siRNA delivery and gene silencing," International Journal of Pharmaceutics, vol. 367, no. 1-2, pp. 195-203, 2009.

[31] J. Du, Y. Sun, Q. S. Shi et al., "Biodegradable nanoparticles of mPEG-PLGA-PLL Triblock Copolymers as Novel Non-Viral Vectors for Improving siRNA Delivery and Gene Silencing," International Journal of Molecular Sciences, vol. 13, no. 1, pp. 516-533, 2012.

[32] T. Lobovkina, G. B. Jacobson, E. Gonzalez-Gonzalez et al., "In vivo sustained release of siRNA from solid lipid nanoparticles," ACS Nano, vol. 5, no. 12, pp. 9977-9983, 2011.

[33] J. Zhou, T. R. Patel, M. Fu, J. P. Bertram, and W. M. Saltzman, "Octa-functional PLGA nanoparticles for targeted and efficient siRNA delivery to tumors," Biomaterials, vol. 33, no. 2, pp. 583-591, 2011.

[34] M. Abbasi, H. Uludağ, V. Incani, C. Y. M. Hsu, and A. Jeffery, "Further investigation of lipid-substituted poly(Llysine) polymers for transfection of human skin fibroblasts," Biomacromolecules, vol. 9, no. 6, pp. 1618-1630, 2008. 

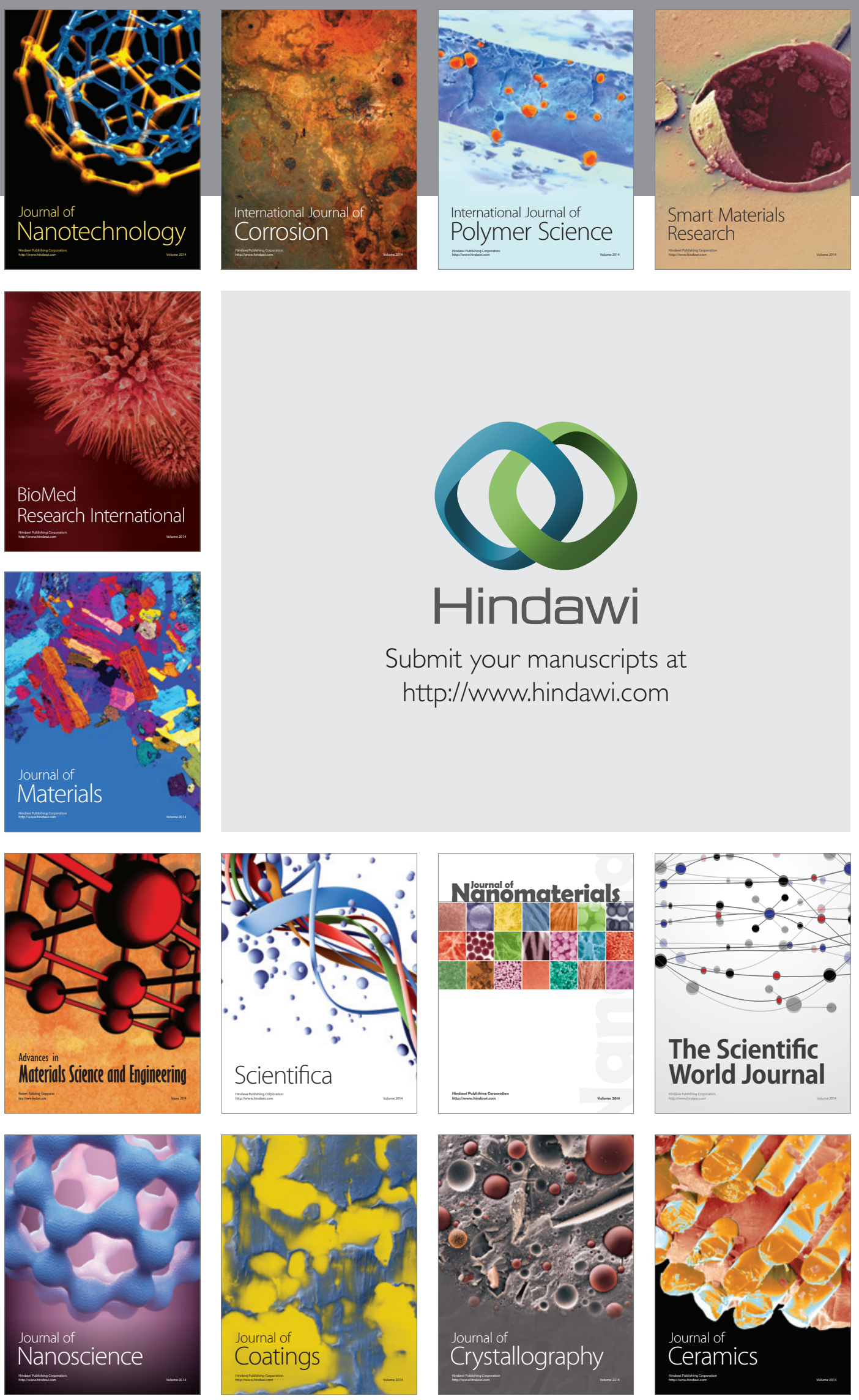

The Scientific World Journal

Submit your manuscripts at

http://www.hindawi.com

\section{World Journal}

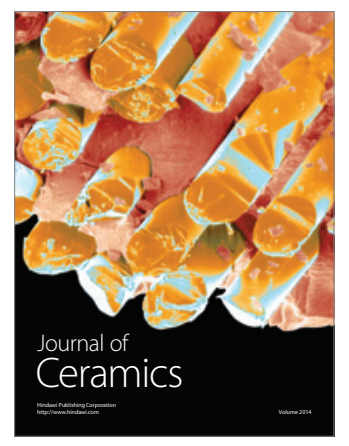

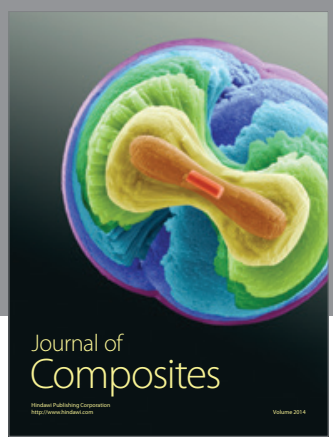
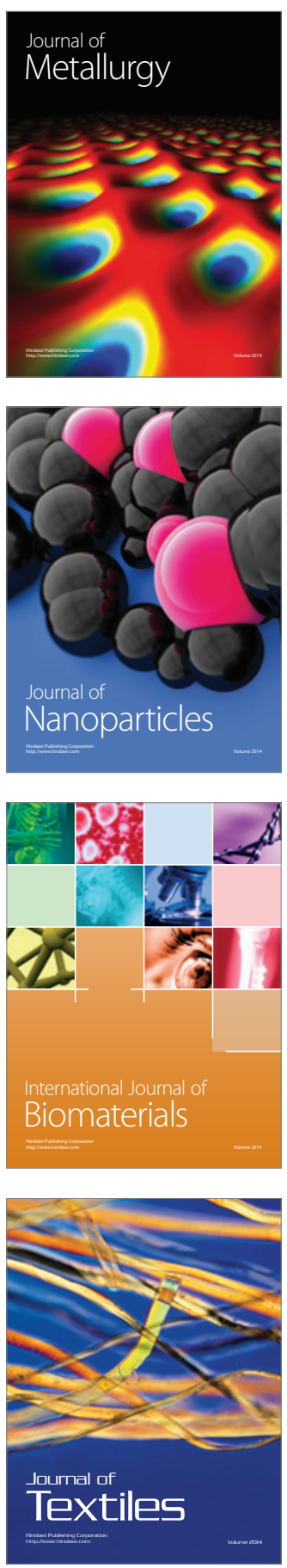